\title{
Individualized ventriculostomy in hydrocephalus: An intravital anatomical study
}

\author{
Martin Scholz, Diane Mielke*, Britta Fricke**, loannis Pechlivanis, Martin Engelhardt, \\ Kirsten Schmieder, Albrecht G. Harders \\ Department of Neurosurgery, Ruhr University Bochum, Knappschaftskrankenhaus, Germany, *Department of Anesthesiology and Intensive \\ Care Medicine, Ruhr University Bochum, Germany, ${ }^{* *}$ Department of Neuroanatomy, Institute of Anatomy, Ruhr University Bochum, Germany
}

\begin{abstract}
Background: Ventriculostomy is a common neuroendoscopic operation but one with disastrous complications in rare cases. Aims: The aim of this study was to perform an intravital analysis of the configuration at the floor of the third ventricle as a possible basis for selection of the ventriculostomy site. Materials and Methods: The study population consisted of 32 patients who underwent ventriculostomy for the treatment of hydrocephalus. Perforation of the floor of the third ventricle was carried out on an individual basis following evaluation of the anatomic situation. Video material and magnetic resonance images (MRI) were analyzed. Results: A classification system including three major groups was developed using the inner distance of the mamillary bodies as the key criterion. It was defined as narrow for values between 0 and $1 \mathrm{~mm}$ (observed range: $0-0.5 \mathrm{~mm}$ ), medium for values between 1.1 and 3.4 $\mathrm{mm}$ (range 1.1-3.4 $\mathrm{mm}$ ) and large for values greater than $3.4 \mathrm{~mm}$ (range: $3.8-6.9 \mathrm{~mm}$ ). Statistical analysis of MR and video measurements revealed a good correlation. The ventriculostomy site was rostral of the mamillary bodies in 23 of the patients $(n=27)$ and sligthly occipital in four. The ventriculostomy site was located more to the left in 22 patients and more to the right in five. Conclusion: As a conclusion the ventriculostomy site has to be chosen in each case following a careful review of all available information. A classification system for the anatomical variations as well as the exact size and site of ventriculostomy should be introduced.
\end{abstract}

Key words: Hydrocephalus, neuroendoscopy, third ventricle, ventriculostomy

The anatomy of the floor of the third ventricle is of increasing importance for neuroendoscopic procedures. Mixter $^{[1]}$ developed a technique for performing third ventriculostomy on the floor of the third ventricle in front of the mamillary bodies as a treatment for hydrocephalus.

During the latter procedure the neurosurgeon has to make an opening in the floor of the third ventricle in order to create a connection between the ventricular system and the subarachnoid space. ${ }^{[2]}$ The proximity of the basilar artery and of the oculomotor nerve and hypothalamic centers has to be borne in mind by any neurosurgeon performing this procedure. ${ }^{[3,4]}$ The midline in front of the mamillary bodies has generally been described as the recommended location for ventriculostomy ${ }^{[5-7]}$ The aim of our study was to perform an intravital analysis of the anatomical variations in this area, to compare these findings with the MRI findings obtained prior to ventriculostomy and with the individually specific ventriculostomy procedure in each patient.

\section{Materials and Methods}

\section{Patients and surgical procedures}

During the last eight years endoscopic ventriculostomy was performed in 32 patients (12 females/20 males, aged 8-78 years, average age 47.6 years) with obstructive hydrocephalus at the Department of Neurosurgery, Ruhr University Bochum. In this prospective study emergency ventriculostomies without video-recording were excluded. A rigid $5.9 \mathrm{~mm}$ neuroendoscope (Type: Camaert, ${ }^{[8]}$ Wolf, Knittlingen, Germany) was used in combination with a special holding device (Magic Arm, Philips, Netherlands).

The site of the third ventriculostomy was chosen in each individual case on the basis of the particular anatomic situation encountered and was normally done in the area of highest translucency in front of the mamillary bodies in safe distance to the basilar artery. In all cases where the basilar artery was not visible during surgery, microdopplersonography (Multidop, 
DWL) was performed intraoperatively [Figure 1]. The region in front of the mamillary bodies was divided into four quadrants which were systematically examined in a clockwise fashion at depths ranging from 0.5 to $3.0 \mathrm{~mm}$. Vascular signals could be obtained from the basilar artery and from the prepontine branches.

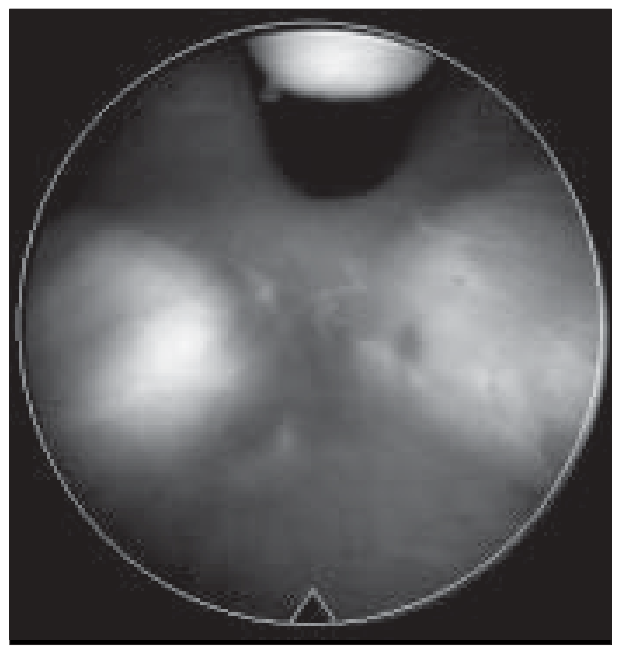

Figure 1: Usage of microdoppler prior to ventriculostomy in a patient with hydrocephalus and aqueductal stenosis
Ventriculostomy was then performed in an area in which no vascular signal could be measured. Following bipolar coagulation with low energy, (Erbotom ICC 350; Erbe Elektromedizin GmbH, Tübingen, Germany combined with a bipolar coagulation fiber, Wolf $\mathrm{GmbH}$ Knittlingen, Germany) perforation and dilation with a Fogarty balloon catheter were carried out (Fogarty Fortis, catheter size 4 French [diameter $1.33 \mathrm{~mm}$ ], balloon maximum size: $9 \mathrm{~mm}$ ). There are two advantages of this technique: 1) in comparison to laser systems the applied energy acts exactly between the bipolar electrodes and not deeper. 2) The transparent balloon of the Fogarty catheter can be opened very slowly under vision. If a minor bleeding from the margin of the stomy occurs, the balloon can be inflated again in order to compress vascular microstructures. The recommended maximum dilation is about $5 \mathrm{~mm}$ in diameter.

\section{Clinical course and outcome}

Patient data like sex, gender, diagnosis, duration of symptoms, kind of preoperative clinical symptoms and outcome can be found in Table 1. In five patients who had undergone shunt insertion several years previously (color-coded blue), ventriculostomy was performed

\begin{tabular}{|c|c|c|c|c|c|c|c|c|c|c|c|c|}
\hline Patient & $\begin{array}{c}\text { Sex } \\
\text { age } \\
\text { (years) }\end{array}$ & Diagnosis & $\begin{array}{l}\text { Duration of } \\
\text { symptoms }\end{array}$ & Headache & Nausea & $\begin{array}{c}\text { Gait } \\
\text { disturbance }\end{array}$ & $\begin{array}{l}\text { Impaired } \\
\text { balance }\end{array}$ & Fatigue & $\begin{array}{c}\text { Memory } \\
\text { problems }\end{array}$ & $\begin{array}{c}\text { Visual } \\
\text { problems }\end{array}$ & Incontinence & $\begin{array}{c}\text { Overall } \\
\text { outcome }\end{array}$ \\
\hline 1 & $\mathrm{~F}, 16$ & AST & $5 \mathrm{~m}$ & ++ & ++ & ++ & ++ & ++ & ++ & & & ++ \\
\hline 2 & $M, 74$ & AST & $>80 \mathrm{~m}$ & + & & + & & & 0 & & & + \\
\hline 3 & $F, 47$ & AST & $30 \mathrm{~m}$ & $(+)$ & & & & & & & & $0(+)$ \\
\hline 4 & $F, 29$ & AST & $3 w$ & 0 & 0 & & & & & & & 0 \\
\hline 5 & M, 27 & AST & $27 y$ & 0 & 0 & 0 & & & & & 0 & $\dagger$ \\
\hline 6 & M, 28 & AST, Astro & $1 \mathrm{~m}$ & ++ & ++ & + & + & ++ & & & & 0 \\
\hline 7 & $\mathrm{~F}, 72$ & AST & $8 \mathrm{~m}$ & + & & $(+)$ & & & & & & 0 \\
\hline 8 & M, 55 & AST & $4-5 \mathrm{~m}$ & + & & + & & & & & & 0 \\
\hline 9 & $M, 61$ & AST & $2 m$ & + & & & & & 0 & & 0 & 0 \\
\hline 10 & $F, 20$ & AST & $12 \mathrm{~m}$ & ++ & & & & & & & & + \\
\hline 11 & $F, 21$ & AST & $24 \mathrm{~m}$ & ++ & & ++ & & & & ++ & & ++ \\
\hline 12 & $M, 62$ & AST & $4 m$ & + & ++ & + & & & & & + & + \\
\hline 13 & $F, 20$ & AST, Astro & $24 \mathrm{~m}$ & + & & & & & & & & + \\
\hline 14 & $M, 39$ & AST & $6 w$ & 0 & & & & & 0 & & & 0 \\
\hline 15 & M, 83 & AST & $24 \mathrm{~m}$ & & & $(+)$ & & & & & & 0 \\
\hline 16 & M, 34 & AST,anomaly & $12 \mathrm{~m}$ & & & + & + & & & & & + \\
\hline 17 & M, 54 & AST? & $5 w$ & + & + & + & & & & & & + \\
\hline 18 & $M, 72$ & $A S T$ & $3 m$ & & & $(+)$ & + & & & & & + \\
\hline 19 & $\mathrm{M}, 30$ & AST,Astro & $3-4 m$ & ++ & & & & & & & & ++ \\
\hline 20 & $M, 8$ & $A S T$ & $2 m$ & + & & + & & ++ & & + & & + \\
\hline 21 & $\mathrm{M}, 51$ & AST & $80 \mathrm{~m}$ & & & + & & & + & & + & + \\
\hline 22 & $F, 66$ & AST & $1 \mathrm{~m}$ & & & + & & & & & & + \\
\hline 23 & $M, 53$ & AST, pinalom & $24 m$ & 0 & & & & 0 & & & & 0 \\
\hline 24 & $F, 41$ & AST & $6 \mathrm{~m}$ & ++ & & ++ & & & & & ++ & ++ \\
\hline 25 & $M, 46$ & $\begin{array}{c}\text { AST, post } \\
\text { trauma }\end{array}$ & unknown & & & & & 0 & & & & 0 \\
\hline 26 & M, 53 & AST & $2-3 m$ & ++ & & & & & & & & + \\
\hline 27 & $\mathrm{~F}, 70$ & AST & $3 \mathrm{~m}$ & & & 0 & & & & & 0 & 0 \\
\hline 28 & M, 62 & AST,astro & $18 \mathrm{~m}$ & 0 & & & & & 0 & & & 0 \\
\hline 29 & M, 39 & AST & $1 \mathrm{~m}$ & + & & + & & & & & & + \\
\hline 30 & M, 78 & AST & $24 \mathrm{~m}$ & & & $(+)$ & & & 0 & & & 0 \\
\hline 31 & $\mathrm{~F}, 62$ & $\begin{array}{c}\text { AST, pinealis } \\
\text { cyst }\end{array}$ & $3 \mathrm{~m}$ & ++ & & + & & & + & & & + \\
\hline 32 & $F, 51$ & AST & $48 \mathrm{~m}$ & 0 & & 0 & & & & & & 0 \\
\hline
\end{tabular}

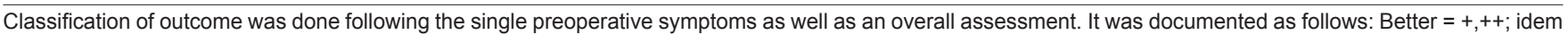

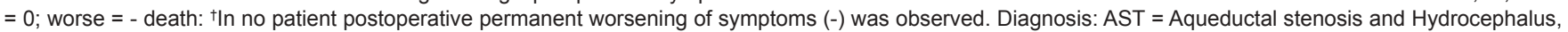

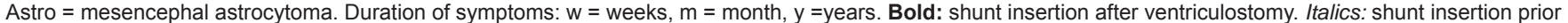
to ventriculostomy 
because of clinical deterioration. In two cases symptoms improved, in the three other cases they did not.

\section{Complications}

No injuries to the basilary tip or prepontine branches occurred in this group; likewise, there were no signs of hypothalamic insufficiency.

There was one death: this 27-year-old male suffered from a hydrocephalus due to aqueductal stenosis (Case 5) with gait disturbance, incontinence and headache and mental deterioration. Because of his aggressiveness to other people and himself he was treated in a psychiatric hospital. After careful evaluation of the case and discussion with the parents who wished the intervention ventriculostomy was performed without problems. The patient was transferred on day 3 back to the Psychiatry. There a cerebrospinal fluid fistula occurred and the young man was sent for revision surgery. This surgery was done in the ambulance and the patient was immediately sent back to the psychiatric clinic. There he died three weeks later due to unknown reasons. We believe the reason was meningitis after re-fistula. Unfortunately, an autopsy was not done.

In five other patients shunt insertion was carried out subsequent to ventriculostomy because of the recurrence of hydrocephalic symptoms, probably as a result of additional communicating hydrocephalus. In three cases the shunt was inserted within the same stay in the hospital, in two other patients four months after ventriculostomy.

There was one other case of CSF fistula (Case 11) which could be managed by local revision, and one mild hemiparesis (4/5) of unknown reason (Case 6) directly postoperative, which disappeared after four days.

\section{Video material}

Video material (S-VHS and DVD) from the patients was analyzed and documented using a high-quality video printer (UP-5600MDP, Sony, Japan). After the first viewing of the video material, morphologic analysis was performed using a checklist including the following items: translucency of the floor of the third ventricle, visualization of the basilar artery, high position of basilary tip, prominence of the mamillary bodies and interbody distance, distance between the mamillary bodies and the infundibulum, visible dorsum sellae and other anatomical details. Prominence of an anatomic structure was chosen as a description for "bulging of a structure over the level of the third ventricle".

The exact location of the ventriculostomy was documented (screen shots). To determine the size and dimensions of the anatomic structures at the floor of the third ventricle, a comparison was made with a $2 \mathrm{~mm}$ bipolar probe placed directly over the interpeduncular fossa in the middle of the endoscopic images. This was done to prevent erroneous measurements caused by the distortion of the endoscopic image. Twenty-nine out of 32 videos were suitable for measurements.

\section{MR examination}

Patients were examined with magnetic resonance tomography (H-SP VB 25A, 1.5 Tesla, Siemens, Erlangen, Germany) in T1- and T2- weighted images with coronal, axial (orbitomeatal) and sagittal sections, $2 \mathrm{~mm}$ slices. The following dimensions and distances were measured in axial, sagittal or coronal sections of the third ventricle: a) in axial sections: width of the right and left mamillary body, length of the right and left mamillary body, inner distance between mamillary bodies, width of the third ventricle; b) in sagittal sections: distance between the infundibulum and mamillar bodies, distance between the aqueduct and optic recess, distance between the mamillar bodies and optic recess, distance between the mamillar bodies and roof of the third ventricle; c) in coronal sections: width of the right and left mamillar body (in relation to visible parts during endoscopy), inner distance between mamillar bodies and distance between the floor and roof of the third ventricle (height of the 3 . ventricle). Figure 2 demonstrates the measured distances in the coronal section of MRI.

The findings of video- and MR-measurements were correlated to clinical data, especially duration of hydrocephalic symptoms. High position of the basilar tip analyzed in the video material means prominence of the basilar tip over the floor of the third ventricle

(visualized by the endoscope). In the MRI measurements we define high position of the basilar tip if the latter is positioned higher than the dorsum sellae.

\section{Anatomic control group}

Four anatomic heads of human specimens from the Anatomical Institute of the Ruhr University Bochum (two females + two males, aged 75-89 years) were

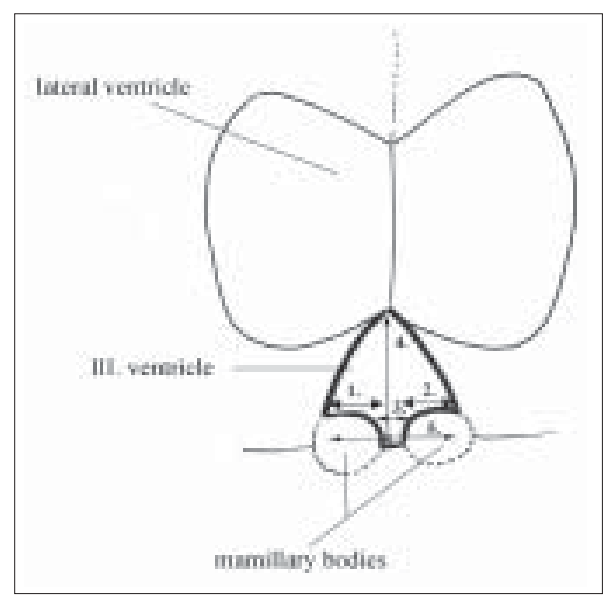

Figure 2: Schematic drawing of a coronal MRI section in the frontal region 1. width of the right mamillary body (in relation to visible parts during endoscopy) 2. width of the left mamillary body 3 . inner distance of mamillary bodies 4 . distance between floor and roof of the third ventricle (i.e., height of the third ventricle) 5 . outer distance of mamillary bodies 
taken as an endoscopic control group. No history of hydrocephalus was known in these individuals. Studies were carried out on these specimens after obtaining the approval of the Ethics Committee of Ruhr University Bochum (No. 844: Date of acceptance for anatomical Study 28.2.97, Date of acceptance for clinical study 20.1.98, No. 2606 for additional features of visual navigation in a clinical study, Date of acceptance 21.09.05). Measurements were made at the floor of the third ventricle using the Visual Navigation (VN) System (Prototype, Bochum, Germany; ${ }^{[9]}$ ), which permits precise touchless measurement of structures inside the ventricular system. This method, in the used software version, needed an additional person at the computer and was therefore not used for patients in this study.

\section{Results}

\section{Morphological description of anatomy following analysis of video material}

The basilar artery was visible in 17 (58.6\%) cases in
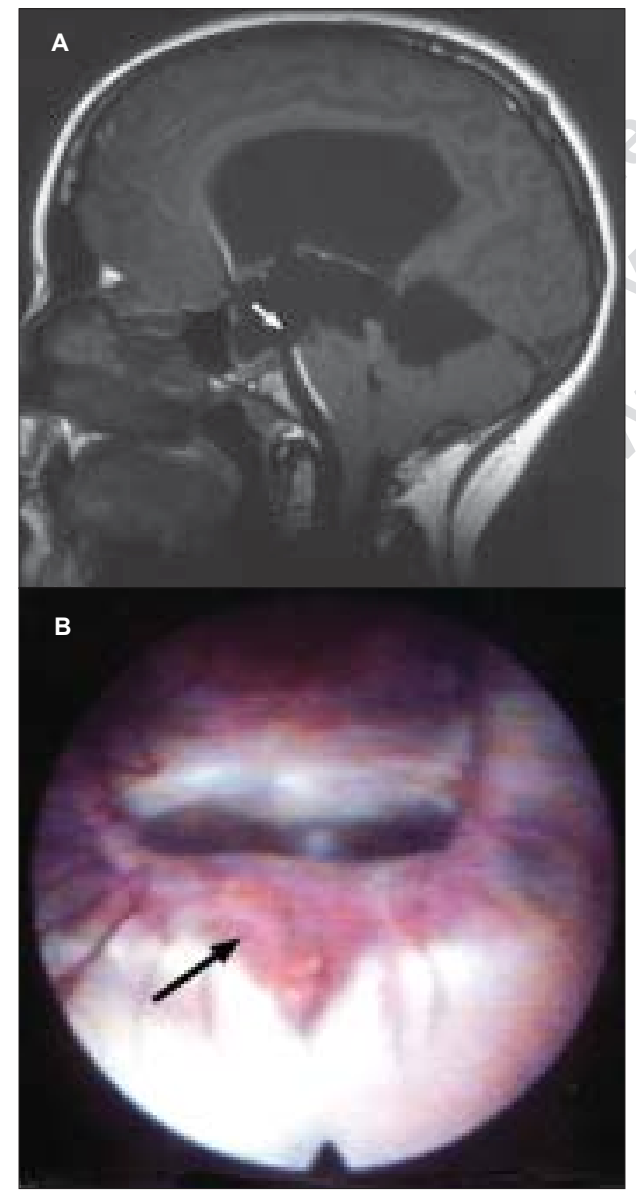

Figure 3: A. Sagittal MRI, T1-weighted images, Pat. no. 1 (16-years-old, F), Group 1a: prominent basilar tip, dorsally excavated third ventricle,

B. Corresponding video print image with a translucent

membrane, narrow inner distance of mamillary bodies, highly positioned basilar tip (arrow) and visible dorsum sellae. the video material $(n=29)$ and was found to be in a very high position in six (20.7\%). Translucency of the membrane over the interpeduncular fossa was found in $23(79.3 \%)$ cases [Figure 3]. Prominence of the mamillary bodies was detected in 11 patients (37.9\%).

Furthermore, other special anatomical variants and situations were observed. The dorsum sellae was visible in two cases and the tuber cinereum [Figure 4] in another two cases with a large distance between the mamillary bodies. In two patients fused mamillary bodies [Figure 5] were noted. The oculomotor nerve was visible in only one case; a complete cavity of all ventricles with a missing foramen of Monro was seen in one patient.

On the basis of the inner distance of mamillary bodies, a classification system with three major groups of anatomic configurations was developed statistically using the distribution of frequency rankings for the observed distances. On the basis of these calculations, the inner distance of mamillary bodies was defined as narrow (Class 1 ) for values between 0 and $1 \mathrm{~mm}$ (0-0.5 $\mathrm{mm}$ found in 11 patients), medium (Class 2 ) for values

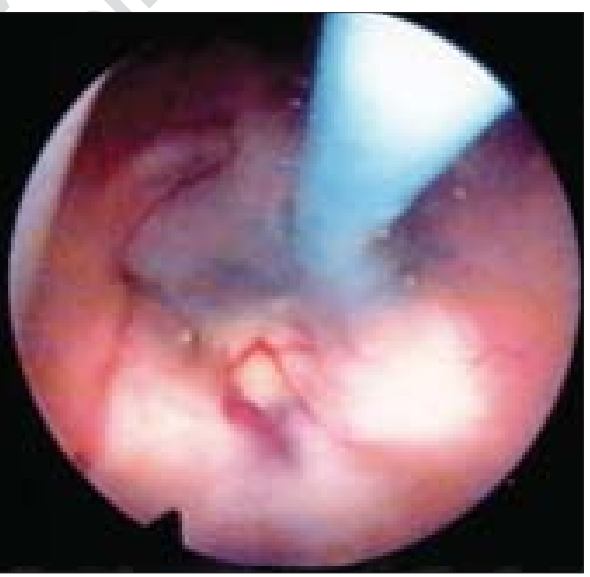

Figure 4: Visible tuber cinereum in a patient with a larger inner distance of mamillary bodies. Coagulation fiber in situ on the right (Case no. 26, 53-year-old man, classification 3b)

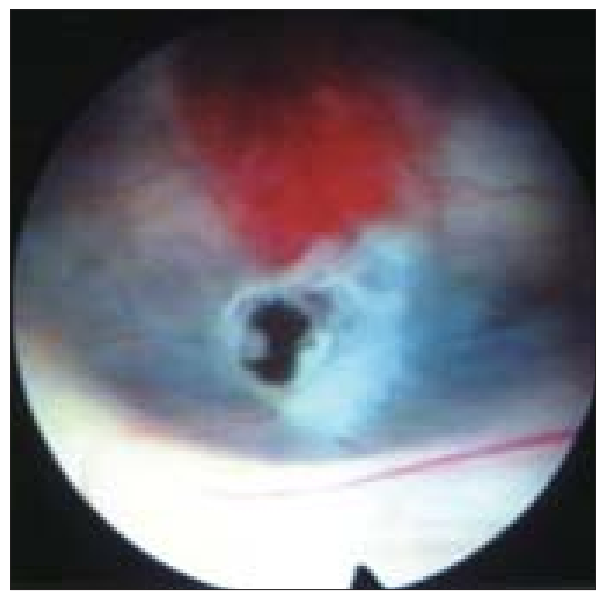

Figure 5: Fused mamillary bodies after ventriculostomy (Patient no. 4, 29-year-old woman, classification 1b). The membrane covering the interperduncular fossa was translucent prior to intervention; the basilar artery is not visible 
between 1.1 and $3.4 \mathrm{~mm}$ (found in 10 patients) and large (Class 3) for values greater than $3.4 \mathrm{~mm}$ (3.8-6.9 mm found in eight patients). Additional anatomic details can be found in Figure 6 and several case examples are presented in Figure 7.

\section{Individual ventriculostomy site}

The ventriculostomy site was rostral and slightly occipital of the mamillary bodies in 23 (85.2\%) and four (14.8\%) cases, respectively. An unusual slightly occipital position between the corpora mamillaria was only chosen in patients with large inner distance of mamillary bodies (Class 3) and translucent membrane behind mamillary bodies but with visible or microdopplerdetected high-positioned basilar artery in front of them. Ventriculostomy site was located more to the left side in

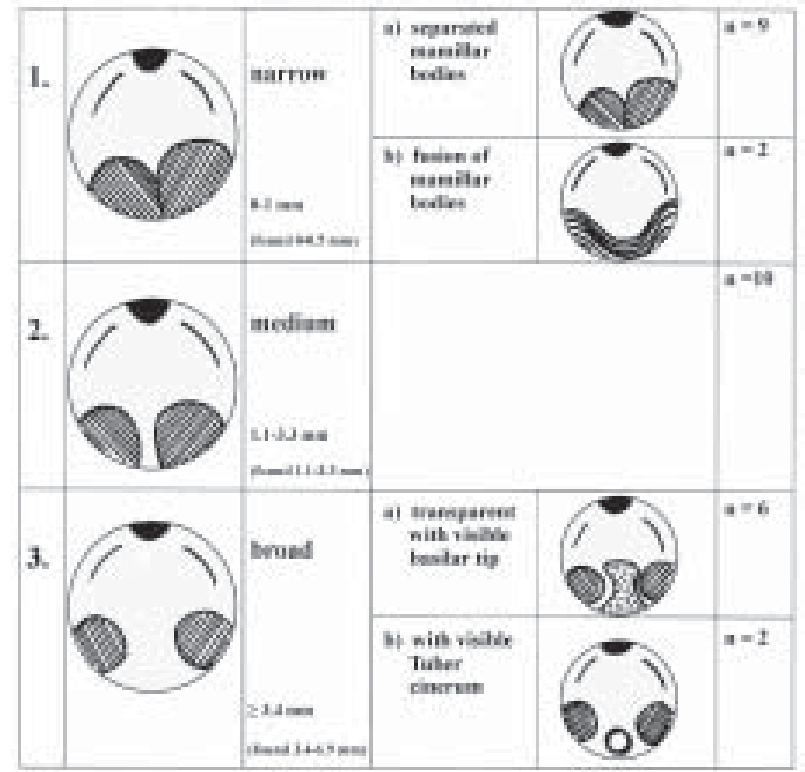

Figure 6: Classification of mamillary bodies in patients with obstructive hydrocephalus (taking consideration of the inner distance)
$22(81.5 \%)$ patients and more to the right side in only five $(18.5 \%)$.

\section{Description of ventricular size and anatomy following MRI}

Table 2 provides an overview of the measured distances for third ventricular anatomy in MRI.

The distances obtained with the video print method were compared statistically with those obtained by MRI. A good correlation of both methods was found with an overall standard deviation of $\pm 1.1 \mathrm{~mm}$. A comparison of the obtained values with standard postmortem values

\begin{tabular}{lcc}
\hline $\begin{array}{c}\text { Table 2: MR measurements of patients with hydrocephalus and } \\
\text { ventriculostomy. All measured distances are given in } \mathbf{~ m m ~}\end{array}$ \\
$\begin{array}{l}\text { Mean and borderline values of measured distances in sagittal } \\
\text { MR sections }\end{array}$ \\
$\begin{array}{l}\text { Distances } \\
\text { Mamillary bodies - infundibulum }\end{array}$ & Mean & Borderline \\
Mamillary bodies - optic recess (middle) & 9.1 & $5.3-18.2$ \\
Recess (middle) - foramen of Monro & 12.8 & $6.3-42.2$ \\
Aqueduct - foramen of Monro & 18.2 & $11.4-26.6$ \\
Aqueduct - recess (middle) & 30.4 & $20.8-45.5$ \\
Width of third ventricle, dorsal part & 34.1 & $24.4-51.1$ \\
Height of third ventricle, rostral part & 16.0 & $10.3-26.6$ \\
Length of mamillary bodies & 17.1 & $7.9-27.3$ \\
in coronal MR -sections & 6.3 & $4.3-12.9$ \\
Width of right mamillary body & & \\
Width of left mamillary body & 6.9 & $4.6-9.7$ \\
Inner distance (mamillary bodies) & 6.3 & $4.2-8.9$ \\
Outer distance (mamillary bodies) & 2.5 & $0-5.2$ \\
Width of third ventricle & 16.2 & $12-19.4$ \\
Height of third ventricle & 16.2 & $10.9-20$ \\
in axial MR sections & 17.6 & $11.4-20.9$ \\
Width of right mamillary body & \\
Width of left mamillary body & 5.7 & $3.0-7.8$ \\
Inner distance (mamillary bodies) & 5.7 & $3.0-10.0$ \\
Outer distance (mamillary bodies) & 3.0 & $0-6.7$ \\
Length of right mamillary body & 14.2 & $9.1-21.3$ \\
Length of left mamillary body & 6.3 & $3.5-11.3$ \\
Width of third ventricle & 6.4 & $4.1-11.6$ \\
\hline
\end{tabular}

Table 2: MR measurements of patients with hydrocephalus and ventriculostomy. All measured distances are given in mm MR sections

Distances

5.3-18.2

6.3-42.2

24.4-51.1

10.3-26.6

$7.9-27.3$

4.6-9.7

$4.2-8.9$

$0-5.2$

12-19.4

$10.9-20$

4-20.9

$0-6.7$

$9.1-21.3$

4.1-11.6

Width of third ventricle
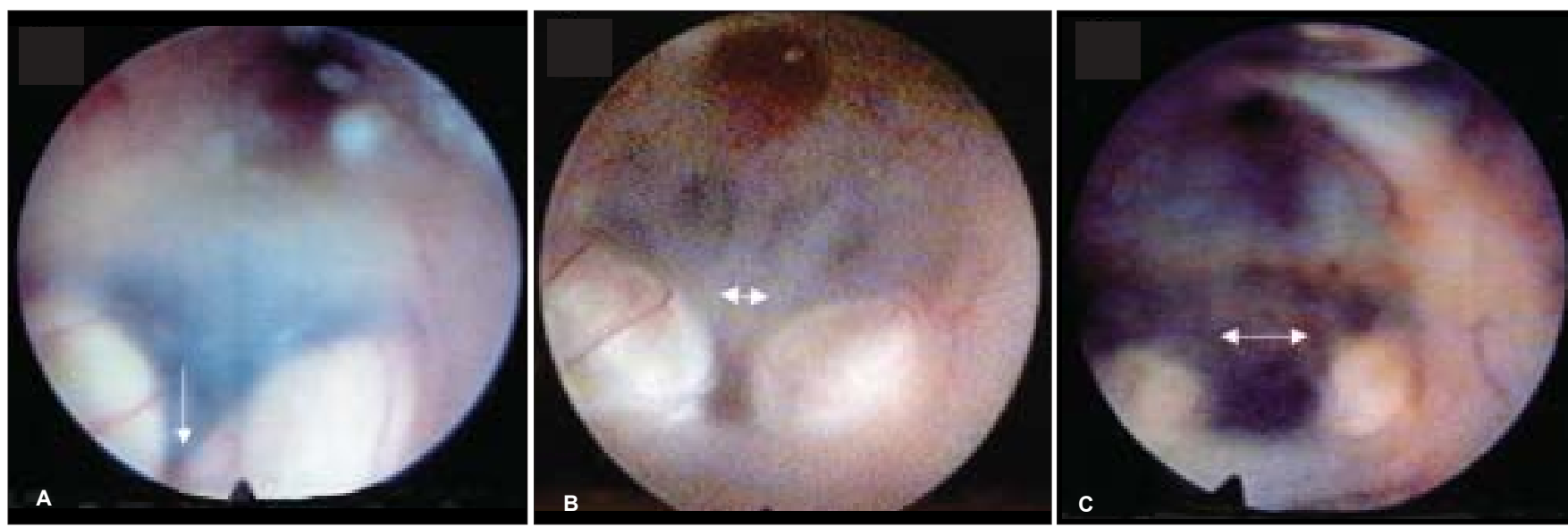

Figure 7: A. Classification of different inner distances of mamillary bodies with video examples: a) Group 1: narrow mamillary bodies (Pat. no. 22, 66-year-old woman). inner distance $=0.4 \mathrm{~mm}$, right mamillary body $=5.3 \mathrm{~mm}$, left mamillary body $=5.7 \mathrm{~mm}, \mathrm{~B}$. Group $2:$ Medium distance of mamillary bodies (Pat. no. 27, 70-year-old woman) inner distance $=1.1 \mathrm{~mm}$, right mamillary body $=5.8 \mathrm{~mm}$ left mamillary body $=6.0 \mathrm{~mm}$, C. Group 3: broad mamillary bodies (Pat. no. 23, 53-year-old man) inner distance $=4.4 \mathrm{~mm}$, right mamillary body $=4.1 \mathrm{~mm}$, left mamillary body $=4.3 \mathrm{~mm}$ 
from the literature ${ }^{[10-12]}$ yielded the following results. The greatest discrepancy in comparison to the control group was noted in the width of the third ventricle. Here the measured values were greater than the values found in the literature by a factor of 2-2.5. Furthermore, the mamillary bodies were 1-2 mm larger than stated in the literature; this discrepancy was noted in both the MRI studies and the video print measurements. The measured values according to the size of the mamillary bodies differed significantly from the values given in the literature. On an average we found a width of $6.39 \mathrm{~mm}$ for the right and $6.09 \mathrm{~mm}$ for the left mamillary body. In the references cited, ${ }^{[10-12]}$ values between 4.0 and 5.0 mm were reported.

The Third Ventricle Index, ${ }^{[13]}$ the quotient between the outer distance of the third ventricle in axial MRI and the width of the brain in axial section, was calculated to be 0.11 . The Evans Index, ${ }^{[14]}$ the quotient between the maximal bifrontal distance of the lateral ventricles and the width of the brain in axial section, was measured at 0.38 ; there was a good correlation between this figure and the hydrocephalus indices given in the literature.

\section{Endoscopic anatomic control group}

The average measurement values obtained are shown in Table 3. An example of such a measurement procedure is given in Figure 8. In all of these cases, the basilar artery was not visible. After a postmortem ventriculostomy procedure the basilary tip was not in a high position. These values correlated well with the measured values reported in the literature. ${ }^{[10,11]}$

\section{Correlations of findings with clinical data}

A correlation between the duration of clinical symptoms and the inner distance of the mamillary bodies was explored. We made a distinction between acute symptomatology (with a duration of up to two months) and chronic disease (duration up to 80 months). The extreme finding of 27 years was excluded from these calculations.

The Pearson correlation coefficient was calculated. A significant positive correlation with a Pearson Index of 0.624 was found for acute symptoms and the inner distance of mamillary bodies. This underlines the

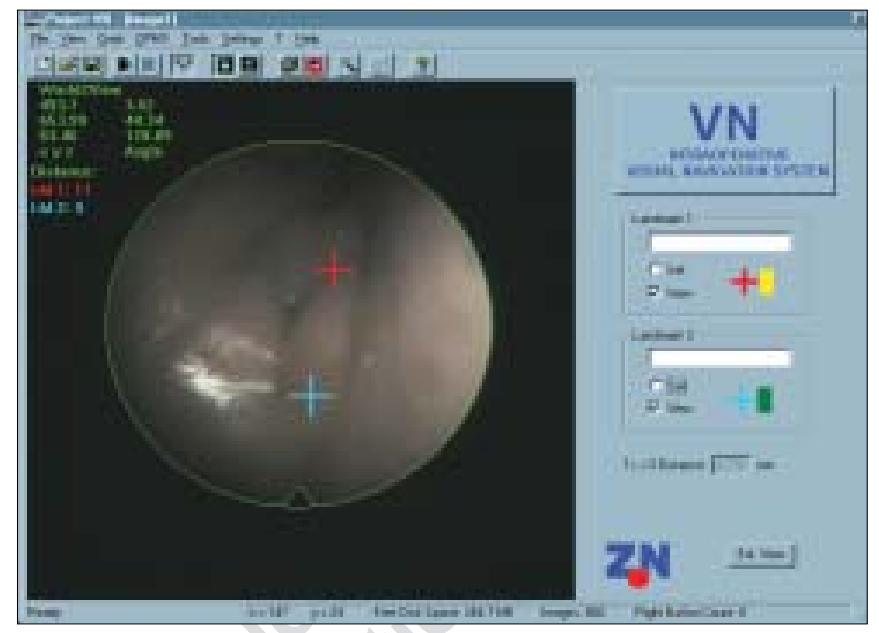

Figure 8: Measurement of the length of the right mamillary body performed on an anatomical specimen with the VN system. The exact distance was $4.8 \mathrm{~mm}$ as shown in the small box on the right below

hypothesis that in acute hydrocephalus the plasticity of the ventricular system under pressure leads to dilations at the weakest points of the ventricular system (e.g., the floor of the third ventricle above the fossa interpeduncularis). As a result side movement of mamillary bodies can be observed. In contrast, no correlation could be obtained between the outer distance of mamillary bodies (i.e., the distance between the outer edges of the mamillary bodies) and the duration of symptoms.

\section{Discussion}

Anatomic relationships in living patients with distinct pathologies are highly relevant for surgical procedures. Unfortunately, postmortem studies performed on pathology specimens can never yield the comprehensive information needed for special surgical questions. Membranes, for example, after formalin fixation, are not translucent in comparison with the intravital situation.

During the past decade several papers were presented dealing with the topographic anatomy under the neuroendoscope. ${ }^{[15-17]}$ In addition, endosonography ${ }^{[18]}$ and conventional sonography ${ }^{[19]}$ were used to obtain

Table 3: Distance measurements in anatomic specimens (normal endoscopic control group)

\begin{tabular}{|c|c|c|c|c|}
\hline Specimen & 1 & 2 & 3 & 4 \\
\hline Age & 85 yrs & 89 yrs & 75 yrs & $87 \mathrm{yrs}$ \\
\hline Gender & $M$ & $\mathrm{~F}$ & $\mathrm{~F}$ & M \\
\hline Inner distance of mamillary bodies & 1 & 0.4 & 1.8 & 0 \\
\hline Outer distance of mamillary bodies & $7.9^{*}$ & $8.8^{*}$ & $7.7^{*}$ & 6.9 \\
\hline Width of right mamillary body & & & & 3.6 \\
\hline Width of left mamillary body & 4.7 & 7.7 & 6.5 & 3.4 \\
\hline Length of right mamillary body & & & & 4.7 \\
\hline Length of left mamillary body & & & & 5.4 \\
\hline Distance infundibulum-mamillary bodies & 11.1 & 11.4 & 13.5 & 7.9 \\
\hline
\end{tabular}


more information intraoperatively. However, the small area in direct proximity to the mamillary bodies, i.e., the target area for ventriculostomy, has seldom been analyzed systematically in living patients until now. ${ }^{[20]}$

The authors have developed a classification system including three major groups using the inner distance of the mamillary bodies as the key criterion. It was defined as narrow for values between 0 and $1 \mathrm{~mm}$ (observed range: 0 - $0.5 \mathrm{~mm}, 11$ patients), medium for values between 1.1 and $3.4 \mathrm{~mm}$ (range 1.1-3.4 mm, 10 patients) and large for values greater than $3.4 \mathrm{~mm}$ (range: $3.8-6.9 \mathrm{~mm}$, eight cases).

Numerous studies dealing with the size of the third ventricle can be found in the literature. ${ }^{[21,22]}$ The comparison of the data collected during our study with the data found in the literature (postmortem specimens) revealed dilation of the ventricular system by a factor of 2; in particular, the distance between the mamillary bodies and the rostral recess was increased. No values for the inner distance of mamillary bodies could be found in the literature prior to this study. ${ }^{[10-12]}$ The fact that the size of the mamillary bodies measured with both methods (MRI and video) was greater by a factor of 2 is interesting. This difference can presumably be explained by increasing dilation of the third ventricle resulting in enhanced prominence of the mamillary bodies, which are normally in the level of the third ventricle. If the mamillary bodies are very distant one may have problems in determining the ideal stomy position at the floor of the third ventricle because the anatomy may be extremely distorted. The floor can also be translucent between the mamillary bodies and the area of possible ventriculostomy may become greater. The inner distance of the mamillary bodies can be used for a classification system for several reasons: 1) it is visible during ventriculostomy, 2) it varies greatly in ventriculostomy patients as was demonstrated in this study and 3) there is a positive correlation between the duration of acute symptoms and the inner distance. The proposed classification scheme offers a useful system for further studies with larger patient populations. Until now, for example, it has remained unclear why patients under the age of two years are at risk for restenosis after ventriculostomy. ${ }^{[23,24]}$ It should be clarified in prospective studies, if there might be a correlation with the size of the ventriculostomy or with the morphology of the third ventricle. It would also be interesting if the morphologic measurements shown in this study can be performed on the postoperative MRI scans following the procedure for comparison, to understand the dynamics of postoperative ventricular plasticity as well.

During ventriculostomy the basilar artery and its branches are of major interest because of their proximity to the area of intervention. We should not overlook the handful of reports of major fatal complications caused by injury to the basilar artery or its branches. ${ }^{[5,6,25,26]}$ Schröder ${ }^{[6]}$ reported the postoperative occurrence of a subarachnoid hemorrhage after ventriculostomy. In another case report McLaughlin ${ }^{[26]}$ described a traumatic basilar artery aneurysm that developed postoperatively in a three-year-old girl with a 'not translucent' floor of the third ventricle.

In the wake of these disastrous cases various proposals have been made for increasing the safety of this procedure. Riegel, ${ }^{[27]}$ for example, describes a combination of interactive neuronavigation and intraoperative fluoroscopy for evaluating patients with a thickened floor of the third ventricle. Schroeder ${ }^{[6]}$ recommends performing ventriculostomy in the midline directly behind the dorsum sellae. He believes that injury to the basilar artery and its branches or third nerve can be avoided by taking this approach.

Vinas $^{[7]}$ and Grant ${ }^{[5]}$ agree with a ventriculostomy in the midline but do not make any stipulations regarding the distance to the mamillary bodies.

In our opinion the dorsum sellae cannot be palpated in every case and injury to the hypothalamic structures is also conceivable when ventriculostomy is performed from the very front of the mamillary bodies. There is no location on the floor of the third ventricle that can be considered generally "the one and only" ideal ventriculostomy point. In contrast, the ventriculostomy site should be chosen on a patient-topatient basis following 1) analysis of MR images and 2) intraoperative analysis of the anatomic situation, including microdopplersonography ${ }^{[19,28-30]}$ if necessary. The latter procedure is especially important in cases where the interpenduncular fossa is covered by a nontranslucent membrane and can detect small prepontine vessels with a diameter under $1 \mathrm{~mm}$.

It is important to mention that ventriculostomy should normally not be performed posteriorly to the mamillary bodies because of the rather high risk of traumatizing the mesencephalus. This can be done right in the middle of mamillar bodies or slightly posterior only in very special cases with an extremely translucent floor of the third ventricle if there is no other place to perform the opening (e.g., with very high-standing thick basilary tip).

\section{Conclusion}

Meticulous analysis of MRI films made preoperatively, intraoperative use of state-of-the-art techniques such as microdopplersonography and an individual approach to ventriculostomy that takes into account any anatomic variants can make endoscopic ventriculostomy a safer procedure. The establishment of a classification system for the anatomical variations at the floor of the third ventricle using measurements as well as the exact documentation of the size and site of ventriculostomy is highly recommended for comparison of results 
from different centers. Classifications using size of the foramen of Monro, the inclination of the floor, the distance between the dorsum and the basilar artery, the position of the basilary tip or the transparency of the floor of the third ventricle etc are also possible and should be analyzed in the future.

\section{References}

1. Mixter $\mathrm{J}$. Ventriculoscopy and puncture of the floor of thethird ventricle. B oston Med Surg J 1923;188:277-8.

2. Hellwig D, Grotenhuis J A, Tirakotai W, Riegel T, Schulte DM, $B$ auer $L B, \mathbb{A}$ al. Endoscopic third ventriculostomy for obstructive hydrocephalus. N eurosurg Rev 2005;28:1-38.

3. Grotenhuis J A. How to avoid complications of endoscopic third ventriculostomy? In: Ferrer $E$, editor. Minimally invasive neurosurgery. Moduzzi E ditore, B ologna; 1999. p. 3-7.

4. Teo C, Rahman S, B oop FA, Cherny B. Complications of endoscopic neurosurgery. Childs N erv Syst 1996;12:248-53.

5. Grant J A, MCL one D G. Third ventriculostomy: A review. Surg N eurol 1997;47:210-2.

6. Schroeder H W, Warzok RW, Assaf J A, Gaab MR. Fatal subarachnoid hemorrhage after endoscopic third ventriculostomy. J Neurosurg 1999;90:153-5.

7. Vinas F C, Castillo C, Diaz F G. Microanatomical B asis for the Third Ventriculostomy. Minim Invas N eurosurg 1996;39:116-21.

8. Caemaert J, Abdullah J , Calliauw L. A multipurpose cerebral endoscope and reflections on technique and instrumentation in endoscopic neurosurgery. Acta N eurochir Suppl 1994;61:49-53.

9. Konen W, Scholz M, Tombrock S. The VN-project: Endoscopic image processing for neurosurgery. Comput Aided Surg 1998;3:144-8.

10. L ang J Stefanec $P, B$ reitenbach W. Form and measurements of thethird ventricle, visual pathway sections and oculomotor nerve. N eurochirurgia (Stuttg) 1983;26:1-5.

11. L ang J. Topographic anatomy of preformed intracranial spaces. Acta N eurochir Suppl (Wien) 1992;54:1-10.

12. Last RJ, Tompsett DH. Casts of the cerebral ventricles. B r J Surg 1939;40:525-43.

13. Oka $K, G o Y, K$ in $Y, U$ tsunomiya $H$, Tomonaga $M$. The radiographic restoration of the ventricular system after third ventriculostomy. M inim Invas N eurosurg 1995;38:158-62.

14. Evans WA. An encephalographic ratio for estimating ventricular enlargement and cerebral atrophy. Arch N eurol Psychiatry 1942;47:931-7.

15. Matula $C$, Tschabitscher $M, K$ itz $K$, Reinprecht $A, K$ oos WT. $N$ euroanatomical details under endoscopical view - relevant for radiosurgery? Acta N eurochirurgica 1995;63:1-4

16. Oka K, GoY, K in Y, Tomonaga M. An observation of the third ventricle under flexible fiber optic ventriculoscope: $\mathrm{N}$ ormal structure. Surg $\mathrm{N}$ eurol 1993;40:273-7.

17. Riegel $T, H$ ellwig $D, B$ auer $B L$, Mennel HD. Endoscopic anatomy of the third ventricle. Acta N eurochir Suppl 1994;61:54-6.

18. Resch K D, Reisch R. E ndo-neuro-sonography: A natomic aspects of the ventricles. Minim I nvas N eurosurg 1997;40:2-7.

19. Rieger A, Rainow N G, Sanchin L, Schopp G, B urkert W. U Itrasoundguided endoscopic fenestration of the third ventricular floor for non-communicating hydrocephalus. Minim Invasive Neurosurg 1996;39:17-20.

20. Rohde V, Gilsbach J M. Anomalies and variants of the endoscopic anatomy for third ventriculostomy. Minim Invasive $\mathrm{N}$ eurosurg 2000;43:111-7.

21. Gyldensted C. Measurements of the normal ventricular system and hemispheric sulci of 100 adults with computed tomography. N euroradiology 1977;14:183-92.

22. Harvey RW. The volume of the ventricles of the brain. Anat Rec 1911;5:301-5.

23. Cohen $A R$. Endoscopic ventricular surgery. Pediatr Neurosurg 1993;19:127-34.

24. M ohanty A, Vasudev MK, Sampath S, Radhesh S, Sastry K olluri VR. Failed endoscopic third ventriculostomy in children: Management options. Pediatr N eurosurg 2002;37:304-9.

25. H andler M, Abbott R, L ee M. A near-fatal complication of endoscopic third ventriculostomy: Case report. N eurosurgery 1994;35:525-8.

26. MCL aughlin, MR, Wahlig J B, K aufmann AM, Albright AL. Traumatic basilar aneurysm after endoscopic third ventriculostomy. N eurosurgery 1997;41:1400-4.

27. Riegel $T$, Alberti $O$, H ellwig D, B ertalanffy $H$. Operative management of third ventriculostomy in cases of thickened, non-translucent third ventricular floor: Technical note. M inim I nvasiveN eurosurg 2001;44:65-9.

28. Auer L M, H olzer P, Ascher PW H eppner F. E ndoscopic neurosurgery. Acta N eurochir (Wien) 1988;90:1-14.

29. Schmidt RH. U se of a microvascular Doppler probe to avoid basilar artery injury during endoscopic third ventriculostomy. J Neurosurg 1999;90:156-9.

30. Zee C, Go J L, L efkowitz M. Advanced imaging of I ntraventricular and Paraventricular L esions I nvolving the Third Ventricle. In: Michael LJ , Apuzzo editor. Surgery of the third ventricle. Williams and Wilkins: 1998. p. 317-21.

Accepted on 11-06-2007

Source of Support: Nil, Conflict of Interest: None declared. 